\title{
Improvement of Chronic Bleeding in the Patient with Unresectable Advanced Gastric Cancer Using the Decoction of Notoginseng Radix - a Case Report
}

\author{
Hyeonjin Jeon ${ }^{1}$, Sora Park ${ }^{2}$, Sookyung Lee ${ }^{2}$ \\ ${ }^{1}$ Department of Clinical Korean Medicine, Graduate School, Kyung Hee University \\ ${ }^{2}$ Cancer Center, Kyung Hee University Hospital at Gangdong, College of Korean Medicine, Kyung Hee University \\ Objectives: A 74-year-old male patient with unresectable advanced gastric cancer (clinical initial stage $\mathrm{T} 3 \mathrm{~N}+$, \\ Borrmann type III) admitted due to gastric bleeding at tumor site. On first admission day, hemoglobin level was \\ $5.7 \mathrm{~g} / \mathrm{dl}$ and performance status was grade 3 according to Eastern Cooperative Oncology Group Performance \\ Status(ECOG-PS). After performing red blood cell transfusion as an emergency treatment, hemoglobin level was \\ increased up to $9.5 \mathrm{~g} / \mathrm{dl}$. However, bleeding of oozing site was continued. For hemostasis, decoction of notoginseng \\ radix (30g/day) was administered since day 7 after admission. The dose was elevated to $40 \mathrm{~g} /$ day after hemoglobin \\ level was decreased to $6.5 \mathrm{~g} / \mathrm{dl}$ on day 11 . Since then, melena was stopped and hemoglobin level was maintained over \\ $9.1 \mathrm{~g} / \mathrm{dl}$. This case shows the hemostasis effect of decoction of notoginseng radix on gastric bleeding in unresectable \\ advanced gastric cancer.
}

Key Words : Stomach Neoplasms, Hemorrhage, Notoginseng Radix

\section{Introduction}

Bleeding is one of the main symptoms of gastric cancer. It is usually appeared as hematemesis or melena. Chronic or massive bleeding can occur anemia. In gastric cancer, hematemesis is reported in $10-15 \%$ of patients and anemia is reported in $1-12 \%$ of patients. ${ }^{1)}$

Bleeding in gastric cancer patients is one of important symptoms to be controlled which was not limited with cancer stage in clinical practice. ${ }^{2)}$ Chronic bleeding can cause anemia, anorexia, dehydration, and hypoalbuminemia which are related to decrease of quality of life. ${ }^{3)}$ Also heavy bleeding can occur hypovolemic shock which can lead to death. Therefore, treatment of bleeding and maintaining hemoglobin level is important issue in gastric cancer.

In western medicine, gastric cancer bleeding is treated by surgery, endoscopy, intravascular embolization or palliative radiotherapy. ${ }^{4}$ Especially palliative radiotherapy is used in unresectable advanced gastric cancer. ${ }^{5)}$ As a systemic intervention, vitamin $\mathrm{K}$, vasopressin, somatostatin analogues, antifibrinolytic agents like tranexamic acid are also used." ${ }^{4)}$ Red blood cell (RBC) transfusion is also needed if hemoglobin level is less than $7 \mathrm{~g} / \mathrm{dl}{ }^{6}$ )

Notoginseng radix is the dried root of Panax

\footnotetext{
- Received : 26 May 2016

- Revised : 17 June 2016

- Accepted : 17 June 2016

- Correspondence to : Sookyung Lee

Cancer Center, Kyung Hee University Medical Center at Gang-dong

892 Dongnam-ro, Gangdong-gu, Seoul, 05278, South Korea

Tel : +82-2-440-6229, Fax : +82-2-440-7287, E-mail : sookyung@khu.ac.kr
} 
notoginseng (Burk) F. H. Chen which was traditionally used to various hemorrhage disease as a medicine for internal and external use. It has a stasis-resolving effect as well as hemostasis effect, ${ }^{7}$ so it can be appropriate to use bleeding which has stagnant blood like cancer bleeding. There was no report about its effect on cancer bleeding, however the study that notoginseng radix powder decreased the bleeding time of gastric disease has been reported in China. ${ }^{8)}$

Therefore, we report this case which shows the hemostasis effect of decoction of notoginseng radix on chronic bleeding in unresectable advanced gastric cancer patient who refused palliative radiotherapy.

\section{Case}

A 74-year-old male patient with advanced gastric cancer was admitted to department of hemato-oncology because of poor oral intake and melena in March 2016. He had diabetes and hypertension since 15 years ago. He had been diagnosed with advanced gastric cancer (clinical stage $\mathrm{T} 3 \mathrm{~N}+$, Borrmann type III) in January 2015. He had been recommended surgery, but his cardiac dysfunction with left anterior descending territory akinesia and 34\% of ejection fraction (EF) did not allow him to undergo surgery. He refused palliative conventional cancer treatment such as chemotherapy and radiotherapy because of age and toxicity. He had been received palliative treatment of Korean medicine combined with supportive conventional medicine. Since February 2016, he had presented poor oral intake accompanied with melena. His hemoglobin level had been decreased to $7.3 \mathrm{mg} / \mathrm{dl}$, so he had received $\mathrm{RBC}$ transfusion over two times. However, his gastric bleeding was continued.

At admission, he presented melena, pale yellowish face, severely poor oral intake and poor performance status of Eastern Cooperative Oncology Group Performance Status (ECOG-PS) 3. His hemoglobin level was decreased to $5.7 \mathrm{~g} / \mathrm{dl}$ and blood pressure measured down to $86 / 50 \mathrm{mmHg}$ was extremely low. $\mathrm{He}$ received transfusion $\mathrm{RBC} 4$ pints and hemoglobin level was elevated to $9.5 \mathrm{~g} / \mathrm{dl}$. Computed tomography (CT) was performed on a day after admission and esophagogastroduodenoscopy (EGD) on 4 days after admission (Figure. 1). In EGD, oozing was shown at gastric tumor site without active bleeding. Surgery was recommended, but his
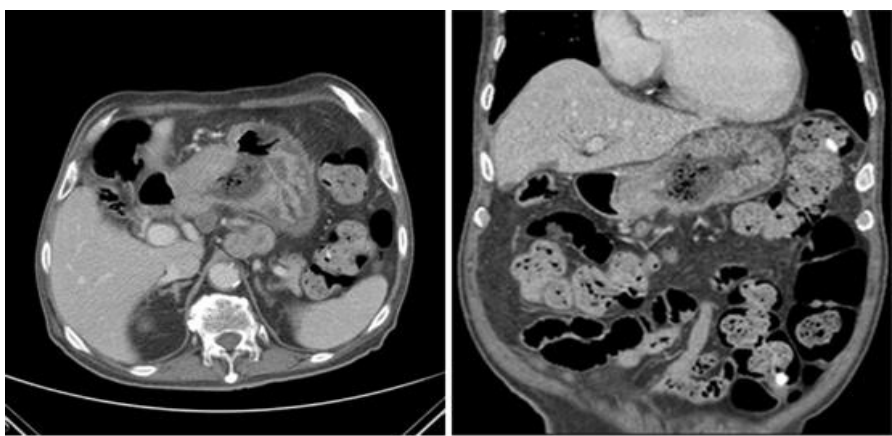

(a)

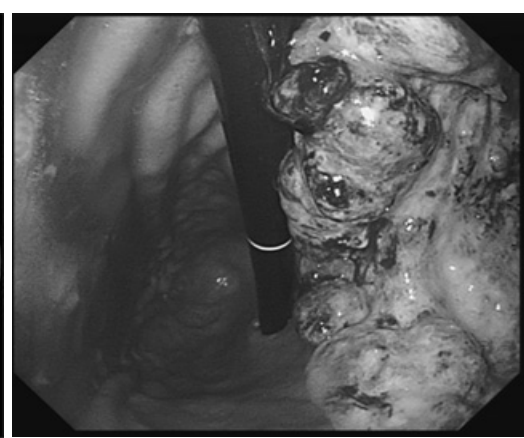

(b)

Fig. 1. The CT scans (a) and esophagogastroduodenoscopy (b).

(a) CT scan was performed on day 1 after admission. Irregular enhancing wall thickening in lesser curvature of low body to antrum with perigastric fat infiltration was observed. (b) Esophagogastroduodenoscopy (EGD) was done on day 4 after admission. The mass of advanced gastric cancer was observed at lesser curvature of middle body to antrum (Borrmann typellI). Ulcerative lesions accompanied by central depression were covered with white coating. Oozing at gastric tumor is observed and there was no evidence of active bleeding. 
Table 1. The Dose of Decoction of Notoginseng Radix

\begin{tabular}{cc}
\hline The day after admission & Amount (g/day) \\
\hline Day 7 to 10 & 30 \\
Day 11 to 35 & 40 \\
Day 36 to 57 & 20 \\
\hline
\end{tabular}

left ventricular dysfunction was too severe to undergo surgery. So palliative radiotherapy was recommended but he refused conventional treatment.

He wanted to receive Korean medicine. On 7days after admission, decoction of notoginseng radix of $30 \mathrm{~g}$ in a day dose was administered three times for the hemostasis treatment. On day 11, the dose was elevated to $40 \mathrm{~g} /$ day because hemoglobin level fell down to $6.5 \mathrm{~g} / \mathrm{dl}$ despite RBC transfusion (Table. 1). At the same time, modified hyeongbangjiwhang-tang was administered (Table. 2). Because he was admitted in hemato-oncology department, he concurrently received tranexamic acid $250 \mathrm{mg}$ twice a day on day 1 to day 6 and once a day during day 7 to day16. RBC transfusion was done on day 1,11 , 12, 15 and 16 (Figure. 2).

During the treatments, hemoglobin level was increased through RBC 4 pints transfusion on day 11 and 12 and maintained more than $10.7 \mathrm{mg} / \mathrm{dl}$. On day 16 , melena also stopped and he discharged.
After the discharge, he visited hospital once a week. Hemoglobin level was maintained more than $9.1 \mathrm{mg} / \mathrm{dl}$. Oral intake and performance also improved and melena did not recur. Therefore the dose of decoction of notoginseng radix was reduced $40 \mathrm{~g}$ /day to $20 \mathrm{~g} /$ day (Table. 1) and the dose of modified hyeongbangjiwhang-tang was also changed (Table. 2). During the treatment, he was also received acupuncture and moxibustion.

As outpatient of hemato-oncology department, the RBC transfusion was performed at day 28 and 50 for the preventive management, intravenous iron therapy as ferric hydroxide sucrose complex 540mg was administered at day 22, 43 and 57, and oral iron (ferrous sulfate dried 256mg) was administered twice a day after day 28(Figure. 2).

\section{Discussion}

In gastric cancer patients, gastric bleeding is one of common symptoms and it can manifest as hematemesis or melena. Furthermore, chronic bleeding can lead to anemia which affect to poor performance status and massive bleeding can occur hypovolemic shock. Therefore, in cancer clinical practice, it is important to manage bleeding and

Table 2. The Dose of Modified Hyeongbangjiwhang-tang

\begin{tabular}{|c|c|c|c|c|c|}
\hline \multirow{2}{*}{ Scientific name } & \multicolumn{5}{|c|}{ Amount(g/day) } \\
\hline & Day7 & Day8 27 & Day28-35 & Day36-42 & Day43-57 \\
\hline Hoelen & 8 & 2.7 & 3.4 & 5.7 & 8 \\
\hline Rehmanniae Radix Preparata & 8 & 2.7 & 3.4 & 5.7 & 8 \\
\hline Corni Fructus & 8 & 2.7 & 3.4 & 5.7 & 8 \\
\hline Alismatis Rhizoma & 8 & 2.7 & 3.4 & 5.7 & 8 \\
\hline Plantaginis Semen & 4 & 1.3 & 1.7 & 2.9 & 4 \\
\hline Araliae Continentalis Radix & 4 & 1.3 & 1.7 & 2.9 & 4 \\
\hline Osterici Radix & 4 & 1.3 & 1.7 & 2.9 & 4 \\
\hline Schizonepetae Spica & 4 & 1.3 & 1.7 & 2.9 & 4 \\
\hline Saposhnikoviae Radix & 4 & 1.3 & 1.7 & 2.9 & 4 \\
\hline Notoginseng radix & 4 & 2.7 & - & - & - \\
\hline Scrophulariae Radix & 6 & 2 & 2.6 & 4.3 & 6 \\
\hline Moutan Cortex Radicis & 4 & 1.3 & 1.7 & 2.9 & 4 \\
\hline Rehmanniae Radix & - & - & 1.7 & 4.3 & 6 \\
\hline
\end{tabular}




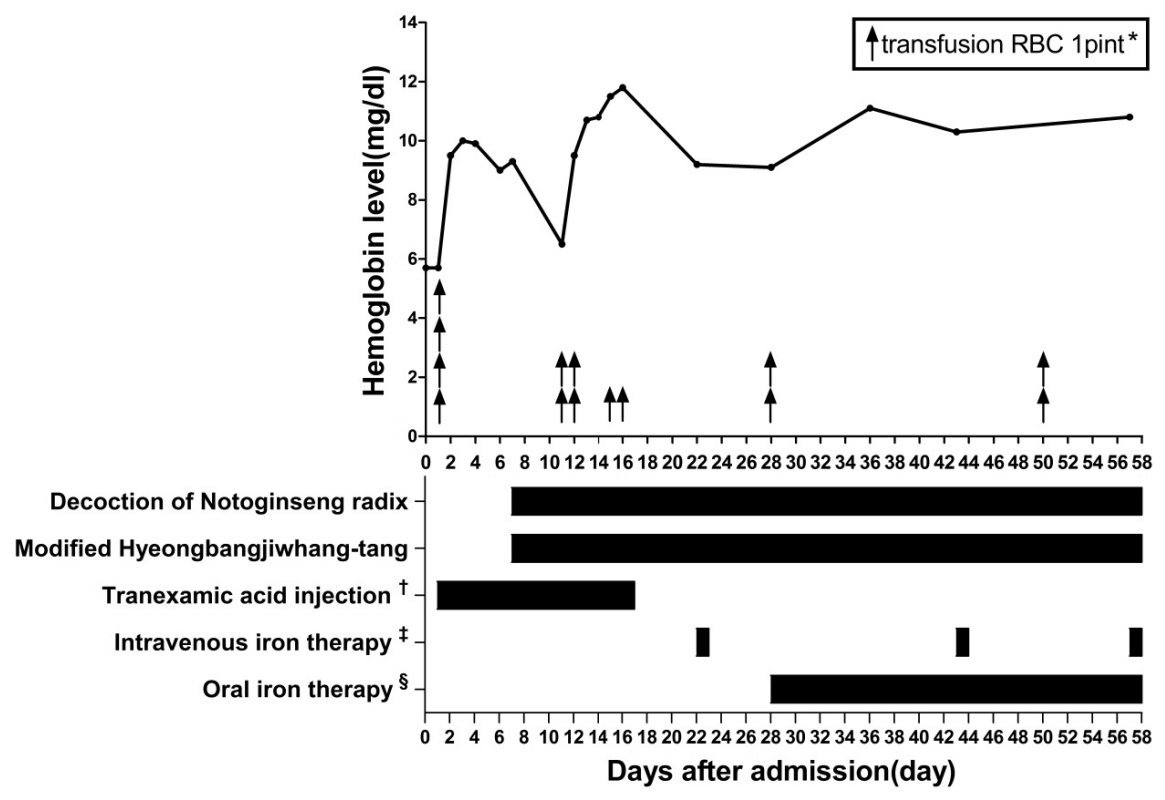

Fig. 2. The hemoglobin level.

*: The volume of RBC transfusion was as follows : 4pint on day 1, 2pint on day 11, 12, 28 and 50. 1pint on day 15 and 16. $†:$ Tranexamic acid was administered as follows : 250mg q12hrs during day 1 to day 6 and $250 \mathrm{mg}$ q24hrs during day7 to day 16 . $\neq$ : Intravenous iron administered at day 22, 43 and 57 as ferric hydroxide sucrose complex $540 \mathrm{mg} \S$ : Oral iron (ferrous sulfate dried 256mg twice a day) was administered after day 28.

correct hemoglobin level. In western medicine, several treatment strategies are used for gastric cancer bleeding such as surgery, endoscopy or intravascular embolization. ${ }^{4}$ National Comprehensive Cancer Network (NCCN) guideline also recommends external beam radiotherapy to gastric cancer bleeding. ${ }^{9)}$ Especially palliative radiotherapy was generally performed to unresectable advanced gastric cancer bleeding. ${ }^{5)}$

This patient visited hospital because of poor oral intake and melena. One year ago, he had been initially diagnosed advanced gastric cancer of clinical stage $\mathrm{T} 3 \mathrm{~N}+$ (Borrman type III). At first admission day, hemoglobin level was decreased down to $5.7 \mathrm{mg} / \mathrm{dl}$, so he received immediately $\mathrm{RBC}$ transfusion. After EGD examination, oozing was found at tumor site, surgery was recommended but he could not undergo it because of severe cardiac dysfunction showing $28 \% \mathrm{EF}$. And he also refused radiotherapy.
The management for gastric bleeding was applied with tranexamic acid, $\mathrm{RBC}$ transfusion and iron supplement therapy at department of hemato-oncology.

However, despite this conventional management including RBC transfusion, his hemoglobin level was decreased to $6.5 \mathrm{~g} / \mathrm{dl}$ showing limitation to stop bleeding.

Tranexamic acid is an antifibrinolytic agent used to reduce hemorrhage but its effect is controversial. Based on the Cochrane review, the effect of tranexamic acid on gastrointestinal bleeding is only shown in the decrease of mortality, however the evidences to protect re-bleeding and to reduce transfusion were limited. ${ }^{10}$ ) Iron therapy is only effective for treating anemia after gastrointestinal bleeding. ${ }^{11)}$ RBC transfusion is used to correct hemoglobin level immediately after bleeding. Furthermore, repeated transfusion is associated with risk of adverse events. ${ }^{12)}$ 
Therefore, decoction of notoginseng radix was administered for hemostasis of oozing site at gastric cancer. Notoginseng radix is one of the stasis -resolving hemostatic herbs in Korean medicine. It has another effect to remove stagnant blood in addition to hemostatic effect. So, it can be apply for bleeding which coexist with stagnant blood $^{7}$, which shows that notoginseng radix has hemostatic effect and anti-hemostatic effect. ${ }^{13)}$ In Korean medicine the pathologic mechanism of cancer is regarded as the association with stagnant blood ${ }^{14)}$.

In herbology, the meridian entries of notoginseng radix are related to liver and stomach. ${ }^{7)}$ Therefore, notoginseng radix looks more suitable for bleeding of gastric cancer than any other herb medicines with hemostatic effect.

One of the main components of notoginseng radix which related hemostatic effect is a dencichine. Several studies reported that dencichine shortened bleeding time through enhancing platelet activation. ${ }^{13)}$ It resulted shorter bleeding time than the extraction powder of raw panax notoginseng, and its hemostatic effect was dependent on the dose. The high dose of dencichine resulted shorter bleeding time and increased platelet aggregation rate. ${ }^{15)}$

In this study, the decoction of notoginseng radix was used for the purpose of convenience to administer easily using small volume. There are some studies to show different effect of notoginseng radix according to administration method. The powder form of ethanol extract revealed the shortest bleeding time as a medicine for external use. ${ }^{13,16)}$ Because the powder can have a hemostasis effect per se, ${ }^{16)}$ the powdered drug can be more effective for external bleeding site. The strong effect of ethanol extracts may be associated with the solubility of major components of notoginseng radix. Dencichine, one of major compounds which identified the hemostatic effect was also effective with oral administration $^{15)}$, and the hydrophilic extract of notoginseng radix also shorten the bleeding time in transected tail of rat. ${ }^{16)}$

During treatment, his hemoglobin level didn't show sharp decline and was maintained over $9.0 \mathrm{~g} / \mathrm{dl}$. Melena was also stopped. And the dose of decoction of notoginseng radix was modified according to the severity of symptom and the level of hemoglobin. This case shows decoction of notoginseng radix could be an effective hemostatic agent for gastric bleeding. However, this case has a limitation which concurrently managed with conventional care. Modified hyeongbangiiwhang-tang, a therapeutic herb for chronic gastrisis $^{17)}$ or digestive trouble ${ }^{18)}$ was administered to treat global symptoms of patient.

Until now, the studies about cancer bleeding in Koran medicine were few and insufficient. There was a case report on rectal cancer bleeding ${ }^{19)}$, and no report on gastric cancer bleeding. Despite of limitation, this case showed the hemostasis effect of decoction of notoginseng radix on gastric bleeding in advanced gastric cancer patient who could not undergo surgery or radiotherapy.

\section{Conclusion}

In conclusion, this case showed the hemostasis effect on gastric bleeding using decoction of notoginseng radix in unresectable advanced gastric cancer.

\section{References}

1. DeVita VT, Lawrence TS, Rosenberg SA. DeVita, Hellman, and Rosenberg's Cancer: Principles \& Practice of Oncology. 10ed. USA: Wolters Kluwer. 2014:615.

2. Maconi G, Manes G, Porro GB. Role of symptoms in diagnosis and outcome of gastric cancer. World J Gastroenterol. 2008;14(8):1149-55.

3. Hashimoto $\mathrm{K}$, Mayahara $\mathrm{H}$, Takashima A, Nakajima TE, Kato K, Hamaguchi $T$, et al. Palliative radiation therapy for hemorrhage of 
unresectable gastric cancer: a single institute experience. J Cancer Res Clin Oncol. 2009; 135(8):1117-23.

4. Pereira J, Phan T. Management of Bleeding in Patients with Advanced Cancer. Oncologist. 2004;9(5):561-70.

5. Kondoh C, Shitara K, Nomura M, Takahari D, Ura T, Tachibana H, et al. Efficacy of palliative radiotherapy for gastric bleeding in patients with unresectable advanced gastric cancer: a retrospective cohort study. BMC Palliat Care. 2015;14:37.

6. Villanueva C, Colomo A, Bosch A, Concepción M, Hernandez-Gea V, Aracil C, et al. Transfusion Strategies for Acute Upper Gastrointestinal Bleeding. N Engl J Med. 2013;368(1):11-21.

7. The co-textbook publishing committee of Korean oriental medicine school. The herbal medicine. Seoul:Younglimsa. 2010:440-1.

8. Wang $\mathrm{Hj}$. The treatment of upper gastrointestinal bleeding using notoginseng powder - 112cases. China Prac Med. 2015;10(6):206-7.

9. Kim MM, Rana V, Janjan NA, Das P, Phan AT, Delclos ME, et al. Clinical benefit of palliative radiation therapy in advanced gastric cancer. Acta Oncol. 2008;47(3):421-7.

10. Bennett C, Klingenberg SL, Langholz E, Gluud LL. Tranexamic acid for upper gastrointestinal bleeding. Cochrane Database Syst Rev. 2014;11: CD006640.

11. Bager P, Dahlerup JF. Randomised clinical trial: oral vs. intravenous iron after upper gastrointestinal haemorrhage - a placebo-controlled study. Aliment Pharmacol Ther. 2014;39(2):176-87.

12. Dasararaju R, Marques MB. Adverse effects of transfusion. Cancer Control. 2015;22(1):16-25.

13. Liang W, Leung PC, Yew DT. Panax notoginseng: A Double-Edged Sword in Haemostasis? Altern Integr Med. 2013;02:e110.

14. Baek MH, Kim DH, Kim SH. The research of East-West medical correlation between cancer and stagnant blood. Journal of istitution of korean medicine in Daejeon University. 1999; 7(2):399-415.

15. Huang LF, Shi HL, Gao B, Wu H, Yang L, Wu $\mathrm{X}-\mathrm{J}$, et al. Decichine enhances hemostasis of activated platelets via AMPA receptors. Thrombosis Research. 2014;133(5):848-54.

16. White CM, Fan C, Song J, Tsikouris JP, Chow M. An Evaluation of the Hemostatic Effects of Hydrophilic, Alcohol, and Lipophilic Extracts of Notoginseng. Pharmacotherapy. 2001;21(7):773-7.

17. Hwang JH, Jang ES, Yoo JH, Kim HS, Lee SW. The Clinical Utilization of Hyungbangiihwang-tang. J Sasang Constitut med. 2008;20(3):142-50.

18. Goo DM. A clinical study on soyangin prescription. J Sasang Constitut med. 2005;17(2):52-63.

19. Lee JY, Choi SH, Park SR, Lee SU, Lee SM, Jung YH. Improvement of cancer bleeding in rectal cancer patient with a herbal decoction, Inhyungtang - A case report. J korean med. 2014;35(4):116-22. 